\title{
Practical Distributed Scheduling for QoS-aware Small Cell mmWave Mesh Backhaul Network
}

\author{
Jiade Li, Yun Zhu, Dapeng Oliver Wu \\ Department of Electrical and Computer Engineering, University of Florida, Gainesville, FL \\ 32611-6130, USA
}

\begin{abstract}
With the explosive growth of mobile data demand, small cells densely deployed underlying the homogeneous macrocells are emerging as a promising candidate for the fifth generation (5G) mobile network. The backhaul communication for small cells poses a significant challenge. However, with huge bandwidth available in the mmWave band, the wireless backhaul at mmWave frequencies can be a promising backhaul solution for small cells. In this paper, we propose Distributed Maximum QoS-aware (DMQ) scheduling algorithm for the mmWave backhaul network of small cells to maximize the system throughput while satisfying QoS requirements for each flow. Based on CSMA/CA, the proposed algorithm is the first that prioritizes MAC contention window to provide better concurrent transmission support while achieving QoS-aware capability. Simulations in the $73 \mathrm{GHz}$ band are conducted to demonstrate $25 \%-40 \%$ performance boost in terms of system throughput and packet loss ratio against other existing schemes.
\end{abstract}

Keywords: Wireless Network; QoS; 5G; mmWave; Directional Antenna.

\section{Introduction}

Some industry and academic experts predict a 1000-fold demand of mobile data increase by 2020 [1]. In order to offer such gigantic demand in data rates and throughput, small cells densely deployed underlying the conventional homogeneous macrocells are emerging as a promising candidate for the fifth generation (5G) mobile broadband [2]. This new network deployment is usually referred to as heterogeneous cellular networks (HCNs). However, with the increase of the number of small cells deployed, the backhaul for small cells becomes a significant challenge $[2,3,4,5]$. Although fiber based backhaul offers large bandwidth, it is costly, inflexible, and time-consuming to connect the densely

Email addresses: jiadeli@ufl.edu (Jiade Li), yunzhu0309@ufl.edu (Yun Zhu), wu@ece.ufl.edu (Dapeng Oliver Wu)

Preprint submitted to Elsevier

July 6, 2016

(C) 2016. This manuscript version is made available under the Elsevier user license http://www.elsevier.com/open-access/userlicense/1.0/ 
deployed small cells. In contrast, wireless backhaul is more cost-effective, flexible, and easier to deploy [3]. With huge bandwidth available, wireless backhaul in mmWave bands, such as the $60 \mathrm{GHz}$ band and E-band $(71-76 \mathrm{GHz}$ and 81-86 GHz), provides several-Gbps data rates and can be a promising backhaul solution for small cells. Meanwhile, rapid progress in mmWave circuits and antennas has accelerated popularization of wireless products and services in mmWave bands [6]. There are also several standards defined in the mmWave bands, such as ECMA-387 [7], IEEE 802.15.3c [8], and IEEE 802.11ad [9].

Unlike existing communication systems using lower carrier frequencies (e.g., from $900 \mathrm{MHz}$ to $5 \mathrm{GHz}$ ), mmWave communications suffer from high propagation loss. To combat severe channel attenuation, directional antennas are utilized at both the transmitter and receiver for high antenna gain. With the beamforming technique, the transmitter and the receiver are able to direct their beams towards each other for the directional communication [10]. The directional communication reduces the interference between links, and concurrent transmissions (spatial reuse) can be exploited to greatly improve network capacity. In a scenario where small cells are densely deployed, effective and efficient backhaul scheduling schemes need to be designed with the characteristics of mmWave communications taken into account.

There has been some related work on the transmission scheduling for mmWave communications. Since time division multiple access (TDMA) is adopted in ECMA-387 [7] and IEEE 802.15.3c [8], many existing works are based on TDMA $[11,12,13,14,15,17]$. One influential work is the Exclusive Region (ER) based scheduling which is introduced and derived in [13]. Qiao et al. [15] proposed a concurrent transmission scheduling considering QoS for flows. In such work, the set of concurrent flows are chosen in a greedy manner to maximize the overall system throughput, through which the number of flows successfully scheduled is maximized.

Although TDMA-based scheduling has been widely adopted in literature, one of its key disadvantage is that a centralized backhaul network controller is needed to coordinate scheduling process. This inevitably increases the system overhead, complicates the system design and limits the scalability of backhaul network. Furthermore, as energy efficiency emerges to be an important metric for next generation wireless networks [16], it also poses a challenge that the increased overhead and complexity would increase the energy cost.

In order to solve the problem and reduce system complexity, there are a few emerging works that propose applying CSMA contention based scheduling on $60 \mathrm{Ghz}$ mmWave band. CSMA has been widely adopted in various existing 802.11 standards and is a practical distributed scheduling framework that does not need centralized control. Gong et al. [21] propose a directional CSMA/CA protocol designed specifically for $60 \mathrm{GHz}$ WPANs. It adopts virtual carrier sensing and relies on a central coordinator to distribute network allocation vector (NAV) information. The authors also extended the work to support spatial reuse in [22]. Similar works can also be found in [23]. Another notable work by Zheng et al. [20] proposes an optimal scheduling algorithm for contention based network although it does not specifically target on mmWave band. However, all 


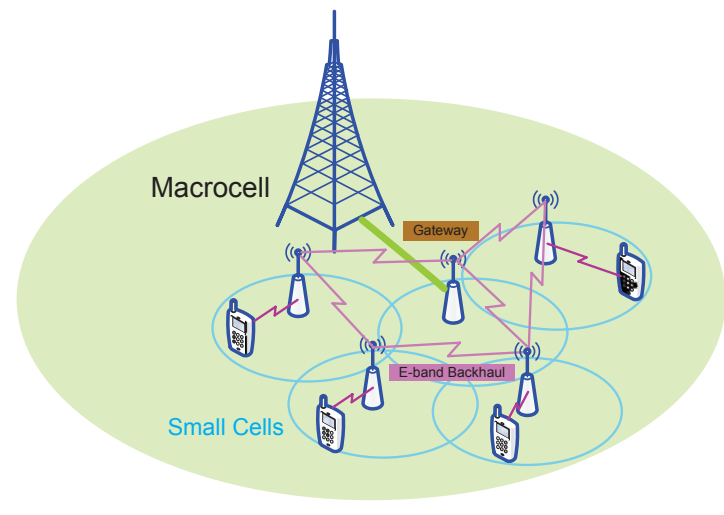

Figure 1: The mesh backhaul network in the small cells densely deployed scenario.

these approaches only target on channel access control and are generally lack of QoS support. Thus they cannot be applied to QoS-aware backhaul networks.

In this paper, we develop a practical CSMA contention based scheduling scheme that is also QoS aware for the small cell backhaul network specifically in mmWave band. The proposed scheme operates in a completely distributed fashion and does not need centralized controller to coordinate flow scheduling. To the best of our knowledge, this is the first CSMA contention based scheduling protocol that prioritizes the MAC contention window to better facilitate both concurrent transmission and QoS support. This greatly reduces the protocol complexity of the network system compared with other existing schemes. Further, we adopt a realistic directional antenna model to evaluate our proposed algorithm for the backhaul network in $73 \mathrm{GHz}$ band. The simulation results demonstrate the superior performance of our algorithm against other existing schemes.

The rest of this paper is structured as follows. Section 2 gives an overview of the system model which includes network model and antenna model. Section 3 formulates the scheduling problem. Section 4 presents in detail our proposed DMQ scheduling algorithm for the mmWave backhaul network. Extensive simulations are conducted and evaluated in Section 5. Finally, Section 6 concludes this paper.

\section{System Model}

\subsection{Network Model}

The characteristic of strong line of sight (LOS) at low inter-site distances (ISDs) of $73 \mathrm{GHz}$ mmWave band generally requires the small cells be deployed densely. Therefore, we consider the scenario where small cells are densely deployed as illustrated in Fig. 1, where we illustrate a typical scenario of densely deployed small cells underlying the macrocell cellular network. In the small 
cells, mobile users are associated with the base stations (BSs), and the BSs are connected via backhaul links with the mesh topology. There are one or more BSs connected to the backbone network via the macrocell site, which are called gateways. Each BS in the network is equipped with an electronically steerable directional antenna, and can direct its beam towards other BSs for directional transmission. The backhaul network is in E-band, which provides high data rates. For the scheduling problem of such backhaul network for small cells, there are two challenges. First, concurrent transmissions need to be fully exploited to maximize the spatial reuse gain. Second, the scheduling scheme should provide the quality of service (QoS) guarantee for flows in the backhaul network. To ensure fairness, the scheduling scheme should maximize the scheduled flows in the network with the QoS requirement of each flow satisfied.

\subsection{Directional Antenna Model}

With BSs fixed, since non-line-of-sight (NLOS) transmissions suffer from higher attenuation than line-of-sight (LOS) transmissions [18], we assume the directional LOS transmission between BSs can be achieved with the locations of BSs adjusted appropriately (e.g., on the roof). Suppose there are $N$ flows requesting transmission, and each flow represents one backhaul link. We denote the distance between the transmitter $s_{i}$ of flow $i$ and the receiver $r_{j}$ of flow $j$ by $d_{i j}$. We also denote the antenna gain of $s_{i}$ in the direction of from $s_{i}$ to $r_{j}$ by $G_{t}(i, j)$, and the antenna gain of $r_{i}$ in the direction of from $s_{j}$ to $r_{i}$ by $G_{r}(j, i)$. Then considering the path loss and signal dispersion over distance, the received power at the receiver $r_{i}$ from $s_{i}$ can be calculated as

$$
P_{r}(i, i)=k_{0} G_{t}(i, i) G_{r}(i, i) d_{i i}^{-n} P_{t}
$$

where $k_{0}$ is a constant coefficient and proportional to $\left(\frac{\lambda}{4 \pi}\right)^{2}(\lambda$ denotes the wavelength), $n$ denotes the path loss exponent, and $P_{t}$ denotes the transmission power $[13,15]$. Due to the half-duplex assumption, adjacent links cannot be scheduled for concurrent transmissions [19]. If flow $i$ and flow $j$ are not adjacent, we denote it by $i \propto j$. Then under concurrent transmissions, the received interference at $r_{i}$ from $s_{j}$ can be calculated as

$$
P_{r}(j, i)=\rho k_{0} G_{t}(j, i) G_{r}(j, i) d_{j i}^{-n} P_{t} .
$$

where we consider $\rho$ as the multi-user interference (MUI) factor related to the cross correlation of signals from different links. Thus, the received SINR at $r_{i}$ can be expressed as

$$
S I N R_{i}=\frac{k_{0} G_{t}(i, i) G_{r}(i, i) d_{i i}^{-n} P_{t}}{N_{0} W+\rho \sum_{j \propto i} k_{0} G_{t}(j, i) G_{r}(j, i) d_{j i}^{-n} P_{t}},
$$

where $W$ is the bandwidth, and $N_{0}$ is the onesided power spectra density of white Gaussian noise [15]. Then according to Shannon's channel capacity, the 
achievable data rate of flow $i$ can be estimated as

$$
R_{i}=\eta W \log _{2}\left(1+\frac{k_{0} G_{t}(i, i) G_{r}(i, i) d_{i i}^{-n} P_{t}}{N_{0} W+\rho \sum_{j \propto i} k_{0} G_{t}(j, i) G_{r}(j, i) d_{j i}^{-n} P_{t}}\right),
$$

where $\eta \in(0,1)$ describes the efficiency of the transceiver design.

For individual antenna, we adopt the reference model described in [26]. First, we define maximum gain of antenna as $G_{0}$, which can be determined by employing the formula of an ideal circular aperture antenna with uniform field distribution:

$$
\begin{aligned}
& G_{0}=(k a)^{2} \\
& k a \cdot \sin \left(\theta_{-3 d B} / 2\right)=1.6162
\end{aligned}
$$

where $k$ and $a$ are the wavenumber and radius of the aperture.

Then, the main-lobe function $G$ is defined as

$$
G(\theta, \phi)=G_{0} \exp \left(-\alpha \theta^{2}\right) .
$$

where $\theta$ is the main-lobe width and $\alpha$ is determined by the half-power beamwidth $\theta_{-3 d B}$ as:

$$
\alpha=\frac{4 \cdot \ln 2}{\theta_{-3 d B}^{2}} .
$$

In unit of $\mathrm{dB}$, the main-lobe can be expressed as:

$$
G(\theta, \phi)[d B]=G_{0}[d B]-3.01 \cdot\left(\frac{2 \theta}{\theta_{-3 d B}}\right)^{2} .
$$

Considering $-20 \mathrm{~dB}$ from the maximum gain as a main-lobe, the main-lobe width $\theta_{m l}$ can be derived from (9) as:

$$
\theta_{m l}=2.6 \cdot \theta_{-3 d B} .
$$

Finally, the side-lobe power $G_{s l}$ can be modeled as:

$$
G_{s l}=\frac{4 \pi-G_{0} \int_{0}^{\theta_{m l} / 2} \exp \left(-\alpha \theta^{2}\right) \cdot \sin \theta d \theta \int_{0}^{2 \pi} d \phi}{\int_{\theta_{m l} / 2}^{\pi} \sin \theta d \theta \int_{0}^{2 \pi} d \phi} .
$$

\section{Problem Formulation}

In this section, we formulate the optimal scheduling problem as a nonlinear integer programming problem.

We assume there is a QoS requirement that represents a minimum throughput requirement for each flow $i$, and denote it by $q_{i}$. We also define a term grouping, which represents a group of nodes that are able to schedule transmissions concurrently. We denote a schedule as $\mathcal{S}$, and assume it has $K$ groupings. 
In each grouping, multiple links are scheduled for concurrent transmissions. For each flow $i$, we define a binary variable $a_{i}^{k}$ to indicate whether flow $i$ is scheduled in the $k$ th grouping. If so, $a_{i}^{k}=1$; otherwise, $a_{i}^{k}=0$. We denote the number of time slots of the $k$ th grouping by $\delta^{k}$.

Since there are different links in different groupings, we denote the transmission rate of flow $i$ in the $k$ th grouping by $R_{i}^{k}$. Then we can obtain $R_{i}^{k}$ as

$$
R_{i}^{k}=\eta W \log _{2}\left(1+\frac{a_{i}^{k} k_{0} G_{t}(i, i) G_{r}(i, i) d_{i i}^{-n} P_{t}}{N_{0} W+\rho \sum_{j} a_{j}^{k} k_{0} G_{t}(j, i) G_{r}(j, i) d_{j i}^{-n} P_{t}}\right) .
$$

Then we can obtain the throughput of flow $i$ based on $\mathcal{S}$ as

$$
T_{i}=\frac{\sum_{k=1}^{K} \delta^{k} \cdot R_{i}^{k} \cdot t_{\text {slot }}}{t_{\text {bo }}+M \cdot t_{\text {slot }}},
$$

where $t_{b o}$ is the time for back-off, and $t_{\text {slot }}$ is the time duration of each time slot on transmission. $M$ denotes the total number of time slot available. Then we define a binary variable $Q_{i}$ to indicate whether the QoS requirement of flow $i$ is satisfied in $\mathcal{S}$. If so, $Q_{i}=1$; otherwise, $Q_{i}=0$. Given the throughput requirements of flows, with limited number of time slots assigned, the optimal schedule should accommodate as many flows as possible [15]. Therefore, the optimal scheduling problem $\mathbf{P}$ can be formulated as follows.

$$
\begin{aligned}
& \text { (P) } \max \sum_{i=1}^{N} Q_{i} \\
& \text { s.t. } \\
& Q_{i}=\left\{\begin{array}{l}
1, \text { if } T_{i} \geq q_{i}, \quad \forall i \\
0, \text { otherwise; }
\end{array}\right. \\
& \sum_{k=1}^{K} \delta^{k} \leq M ; \\
& a_{i}^{k}+a_{j}^{k} \leq 1, \text { if flow } i \text { and } j \text { are adjacent; } \forall i, j
\end{aligned}
$$

It is obvious that this is a nonlinear integer programming problem, and is NP-hard. Constraint (15) indicates if the throughput of flow $i$ in the schedule is larger than or equal to its throughput requirement, $Q_{i}=1$; otherwise, $Q_{i}=$ 0 . Constraint (16) indicates there are at most $M$ time slots. Constraint (17) indicates due to the half-duplex operation of BSs, adjacent links cannot be scheduled for concurrent transmissions since there is at most one connection for each node [19].

Although it is difficult to solve the problem $\mathbf{P}$ in polynomial time, we propose an efficient and practical distributed scheduling algorithm to achieve a suboptimal solution instead in the next section. 


\section{Distributed Scheduling Algorithm}

In this section, we propose a practical Distributed Maximum QoS-aware (DMQ) scheduling algorithm to solve the scheduling problem in (14).

\subsection{Neighborhood Detection}

In order to effectively schedule flows between base stations, each station needs to keep track of how many other stations that can potentially cause interference to the station. One of the advantage of mmWave band is its strong capability for channel reuse. However, interference plays an important role in reducing such channel reuse capability. By detecting information of neighbors, we are able to reduce such interference. One straight-forward way to conduct neighborhood detection could be that every node periodically broadcasts beacon signal to its neighbors. If the other node is able to receive such beacon signal, then it knows it has one neighbor that could possibly in the interference range. However, since interference only occurs when nodes are transmitting, such active signaling mechanism can cause unnecessary overhead over time. The increased overhead will not only impact the performance of the network, but it also increases the energy consumption. We can eliminate such overhead by setting up an overhearing mechanism at each base station.

The neighborhood detection algorithm works as follows. At time slot $t$, each base station $b_{i}$ counts the number of distinct sources for flows not destined to $b_{i}$ by letting the NIC operate in promiscuous mode. Therefore, when a nearby base station $b_{j}(j \neq i)$ sends a packet, base station $b_{i}$ is able to detect the signal and effectively count $b_{j}$ as its neighbor. The detailed procedure is described in Algorithm 1.

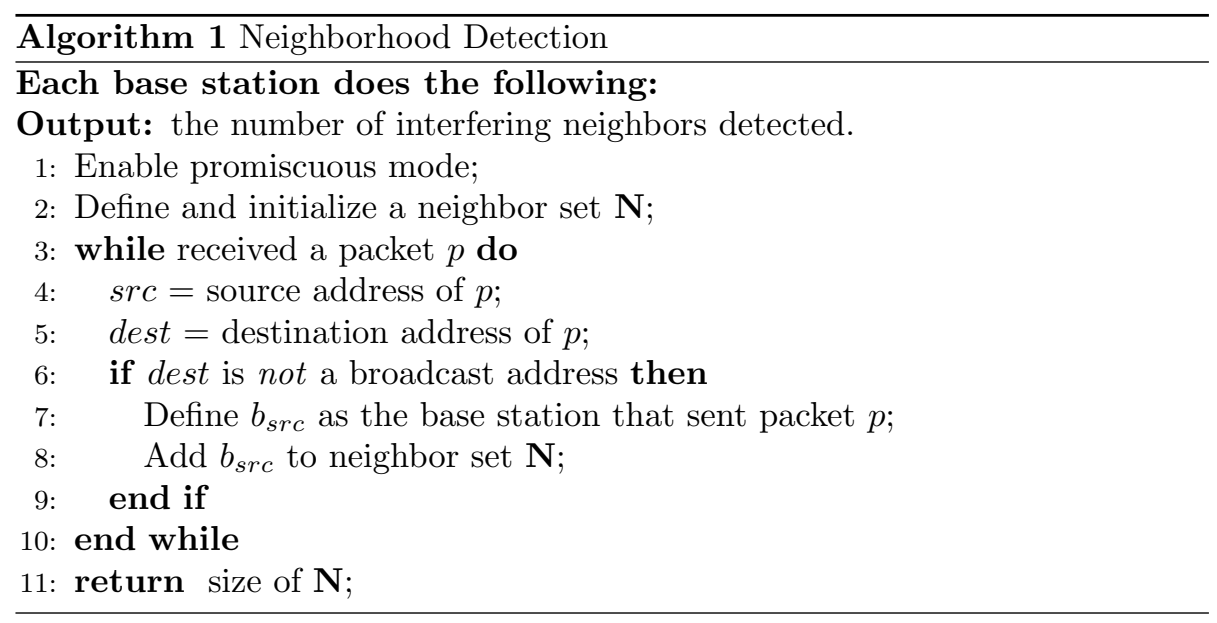

While signal in mmWave band is more concentrated and less likely that it will cause interference in large range, yet it is still not uncommon that interference does happen when several base stations are located in densely deployed small 
cells and, in the meantime, the directional antennas' orientation is also set within the interfering sidelobe or mainlobe area. Thus, for a base station $b_{i}$, there could be simultaneously several neighbor base stations contending for the same channel. This will cause the degradation of network throughput if we don't take measures to mitigate the problem. Our proposed DMQ is right designed for this task. DMQ scheduler is able to schedule flows according to a base station's contention level, which is positively proportional to its number of neighbors.

\subsection{Contention Window Prioritization}

In order to facilitate both contention avoidance and QoS-aware scheduling between base stations, we define eight priority levels indexed from 0 to 7 . For practical concern, DMQ algorithm exploits the Quality of Service (QoS) feature shipped with 802.11e. IEEE 802.11e standard is an amendment to IEEE 802.11 standard family that defines a set of QoS facilitating features, of which the most notable is the Enhanced Distributed Channel Access (EDCA) mechanism. EDCA provides levels of priority which are called Access Categories (ACs). By default, there are 8 distinct ACs indexed from 0 to 7 . We follow such settings in order to make our proposed scheme adopt to 802.11e standard. Each AC has a pair of minimum contention window size (CWmin), maximum contention window size $(\mathrm{CWmax})$. These parameters regulate the back-off behavior of current distributed coordination function (DCF). The larger index number represents higher priority level and vice-versa. Each priority level has parameters of minimum contention window size (CWmin) and maximum contention window size (CWmax). The configuration of these parameters are shown in Table 1.

For a specific priority, the CWmin and CWmax regulate the back-off behavior of current contending base station. When contention occurs, similar to CSMA/CA mechanism, the scheduler will initialize a random back-off window of size within CWmin and CWmax. If contention still occurs after back-off, the scheduler will double the size of current back-off window until it reaches CWmax and will keep it at CWmax until reset. The back-off procedure continues. Generally, the average size of back-off window determines how contentious current base station is. The larger the size of back-off window, the longer the base station will wait to try to initiate next transmission, thus exhibiting less contentious behavior, and vice-versa.

Therefore, we utilize this fact to configure the CWmin and CWmax parameters with respect to their associated priority levels. For contention resolving purposes, we want the base stations operating in lower priority level to be less contentious. Thus we assign them with relatively larger CWmin and CWmax values. Vice-versa, the base stations with higher priority levels will be assigned with smaller CWmin and CWmax values. Furthermore, we also try to adjust CWmin and CWmax to make each priority level more distinct and disparate from one another; such that, for a given priority level $i$, the probability that the randomized back-off window size will overlap with that of priority level $j(j \neq i)$ will be reduced to minimum. Table 1 shows the exact configuration of these parameters, where we completely eliminate the possible back-off window size overlapping between different priority levels. 
Table 1: Contention Window Prioritization

\begin{tabular}{|c||c|c|c|c|c|c|c|c|}
\hline Priority & 0 & 1 & 2 & 3 & 4 & 5 & 6 & 7 \\
\hline CWmin & 512 & 256 & 128 & 64 & 32 & 16 & 8 & 3 \\
\hline CWmax & 1023 & 511 & 255 & 127 & 63 & 31 & 15 & 7 \\
\hline
\end{tabular}

\subsection{Coarse-to-Fine Window Mapping Algorithm}

Based upon prioritized contention window configuration in Table 1, we propose a Coarse-to-Fine Window Mapping (CFWM) algorithm. Namely, CFWM is a two-phase process. In the coarse-phase, the algorithm addresses the contention issue while in the fine-phase, QoS requirements are accommodated.

\subsubsection{Coarse Phase}

Let's first define contention degree $d_{c}^{i}$, as the number of neighboring base stations a base station $b_{i}$ detects. The value of $d_{c}^{i}$ will be calculated by Algorithm 1 described in Section 4.1. CFWM works by assigning priority level to current schedule according to its base station $b_{i}$ 's contention degree $d_{c}^{i}$. We call this coarse phase of CFWM, which is illustrated in Algorithm 2.

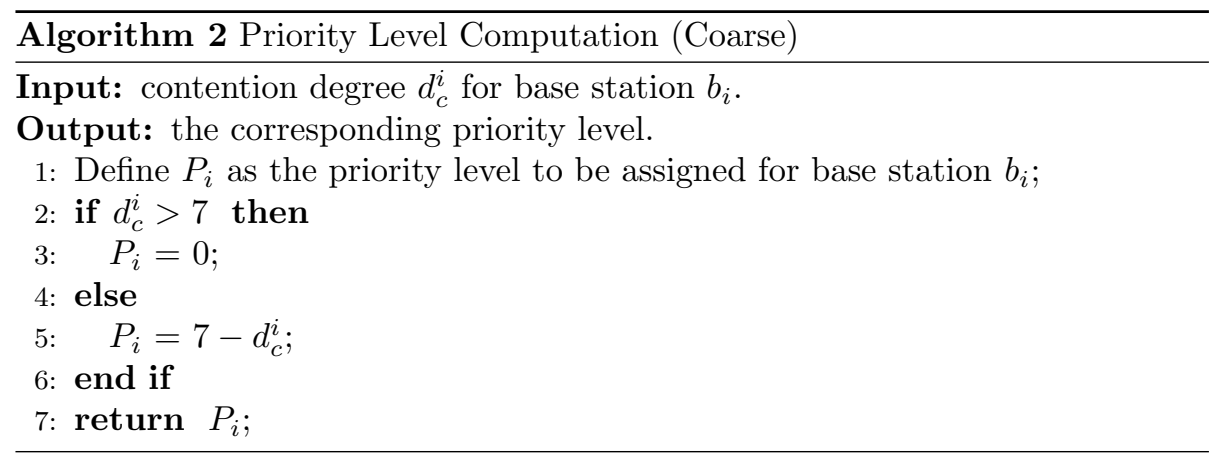

The intuition behind Algorithm 2 is that the scheduler always tries to assign higher priority level to base station $b_{i}$ that has smaller value of contention degree $d_{c}^{i}$. In such case, since the base station has less number of neighbors contending with it (smaller value of contention degree), it is more likely that this base station is able to fulfill the QoS requirement and increase the overall network throughput of the system. Thus, CFWM will assign it with a higher priority level. On the other hand, if base station $b_{i}$ has a larger value of contention degree, the CFWM will assign it with a lower priority level which tends to curb the sending rate of base station $b_{i}$. In practice, the number of contention neighbors a base station potentially has usually would not exceed 7 , thus we believe the linear mapping between contention degree and priority level (line $2-5$ in Algorithm 2) is reasonable. For cases where contention degree does exceed 7, CFWM just assigns priority level 0 , the lowest, to the corresponding base stations. 
In essence, each priority level determines the contention window adjustment strategy. Since the lower the priority level is, the larger CWmin/CWmax will be set, which leads to a less contentious base station. It is also true vise-versa. Our rationale behind this mechanism is that we always want the least contending base station to transmit first, because the less contending a base station is, the higher throughput the base station can potentially create. As will be seen in Section 5, our experimental results also support this rationale.

\subsubsection{Fine Phase}

So far, the priority level assignment procedure described in Algorithm 2 only considers the contention between base stations. The more neighbors a base station $b_{i}$ has, the more contending $b_{i}$ becomes, the lower priority level $b_{i}$ will be assigned. Another important issue that we need to address is QoS-awareness of the DMQ scheduler. On top of the scheduling scheme in Algorithm 2, we equip DMQ with QoS scheduling capability which is described in Algorithm 3. We call this fine phase of CFWM.

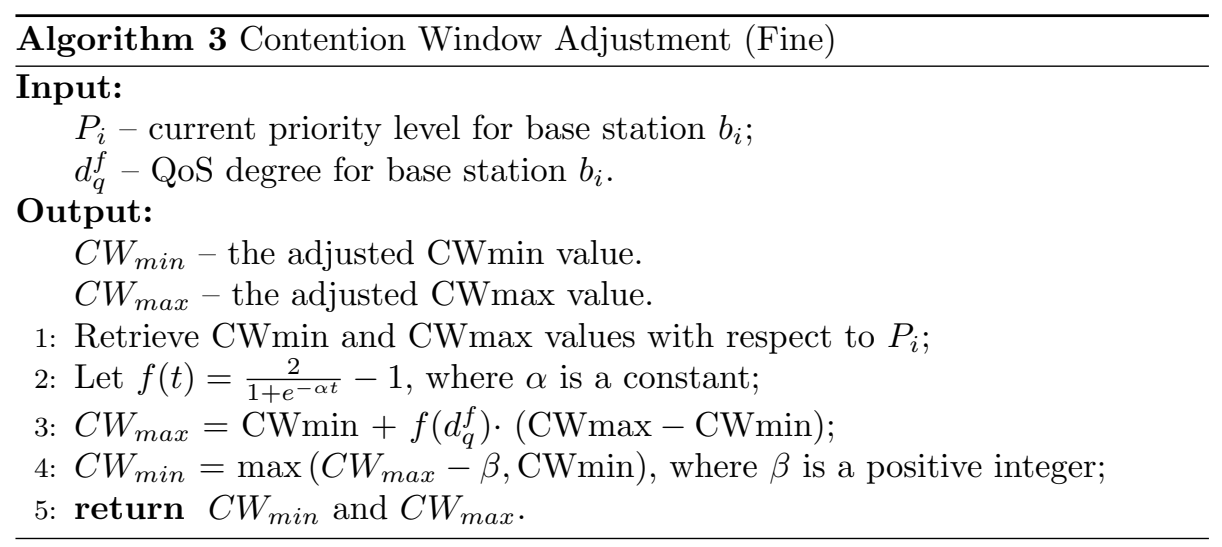

For each flow $f$, define $Q o S$ degree $d_{q}^{f}$ as a positive value that indicates the QoS priority for the flow. The higher the QoS priority is, the larger $d_{q}^{f}$ is assigned. In Algorithm 3, CFWM tries to make a finer contention window parameter adjustment based on $Q o S$ degree $d_{q}^{f}$ for current flow $f$ on base station $b_{i}$. According to our QoS-aware schedule policy, the higher QoS priority a flow has, the sooner it needs to be scheduled in order to achieve optimal throughput over the network. CFWM achieves this purpose by adjusting the CWmin and CWmax value according to the QoS priority inside current priority level $P_{i}$. Recall in Section 4.2, we described that CWmin and CWmax determine the range within which the size of contention window could be. Although not guaranteed, statistically, when CWmin or CWmax increases, the back-off time for current DCF will become longer since the probability to randomize a larger back-off slot number becomes higher. Therefore, when a flow with higher QoS priority needs to be scheduled, Algorithm 3 will return a decreased CWmax and CWmin value (line 3 and line 4 of Algorithm 3) which essentially leads to 
a shorter back-off time. This will make the flow be able to have much larger probability to be scheduled earlier than its contending counterparts if there are any.

Algorithm 2 and Algorithm 3 altogether constitute the cornerstone of our proposed CFWM algorithm. They work cooperatively to schedule flows according to both their contention degree and QoS degree. When a base station $b_{i}$ has a flow to send, CFWM will first retrieve appropriate priority level by referring to Algorithm 2 with contention degree information. Afterwards, CFWM will continue to run Algorithm 3 to set proper CWmin and CWmax values for scheduler's back-off window inside current priority level. Results returned from Algorithm 2 only indicates which priority level current flow should be associated with, while Algorithm 3 further deals with the exact values of CWmin and CWmax inside that priority level. Thus Alogrithm 2 is called coarse phase while Algorithm 3 is called fine phase. That is how CFWM deals with both contention issue and QoS-awareness in a unified framework.

\section{Simulation Results}

In this section, we implemented our proposed DMQ algorithm and compared the performance with existing schemes.

\subsection{Development of Simulator}

For performance evaluation of DMQ, we consider a backhaul network consisted of 25 base stations (nodes) evenly distributed over a 500 meters $\times 500$ meters grid area, as illustrated in Figure 2. Each base station is equipped with directional antennas as well as quasi omni-directional antennas. For each node, we implemented the widely adopted directional antenna model as described in [26]. The beamwidth of the directional antennas is set to 30 degrees and the channel is set to work on $73 \mathrm{GHz}$ band. On top of directional antenna model, our proposed DMQ scheduling algorithm is implemented. The simulation system is developed under NS-3 [27].

For each base station, it is able to communicate with its 8 adjacent neighbors in one-hop distance. We note that for base stations that are out of one-hop distance, an appropriate routing protocol is needed to route packets through multiple hops. Since routing is not our focus in this work, we believe many existing wireless routing protocols should work with our experiment. For simplicity, we just use Optimized Link State Routing (OLSR) protocol to achieve such purpose.

For performance comparison, we also implemented the optimal Distributed Opportunistic Scheduling (DOS) scheme proposed in [20] and a default 802.11 random access scheme as a baseline. To evaluate our proposed scheme, the following metrics are considered:

- System throughput: Needless to say, system throughput is a key performance metric we primarily concern about. By throughput, we mean the overall throughput on the whole backhaul network. We will study 


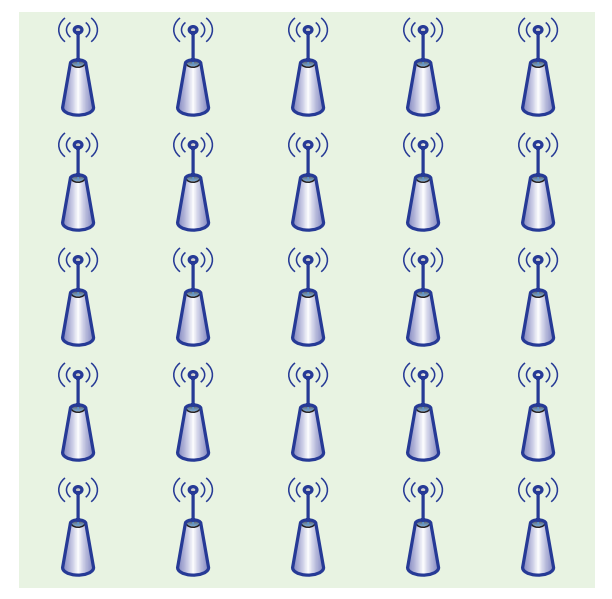

Figure 2: Topology of base stations used in simulation.

the effects of number of flows, effects of number of packets and effects of antenna beamwidth on throughput performance.

- Packet loss ratio: Since we also consider QoS as an essential part of our scheduling, packet loss ratio is yet another important indicator that measures the reliability performance of the scheduling system from a packet level perspective. We will study the effects of number of flows on packet loss ratio.

\subsection{Performance Evaluation}

In this subsection, we design experiments to study the scheduling algorithm performance under different number of flows, under different number of packets in each flow and under different antenna beamwidth. In order to focus on scheduling itself rather than the effect of routing, we use the term "flow" to only refer to the case where the flow is originated at one base station and travels to another in one hop. If there are more than one hop along the path of the flow, e.g. $k(k>1)$ hops, we regard this as $k$ flows. To reduce random error for each experiment, we repeat the same experiment for 10 times and the average results are calculated and taken.

\subsubsection{Effects of Number of Flows on Throughput Performance}

First, we set that each flow is consisted of 10 packets and each packet is of size 1000 bytes. Then, we add flows to the system. The number of flows is varied in range of 10 to 100. Once the number of flows is determined, these flows are generated and added randomly among the base stations. For each base station, one packet is scheduled to be generated and sent from transport layer in every 30 milliseconds, i.e., the sending interval. On the MAC layer, we configured each base station and installed scheduling scheme using, respectively, DMQ, DOS as 


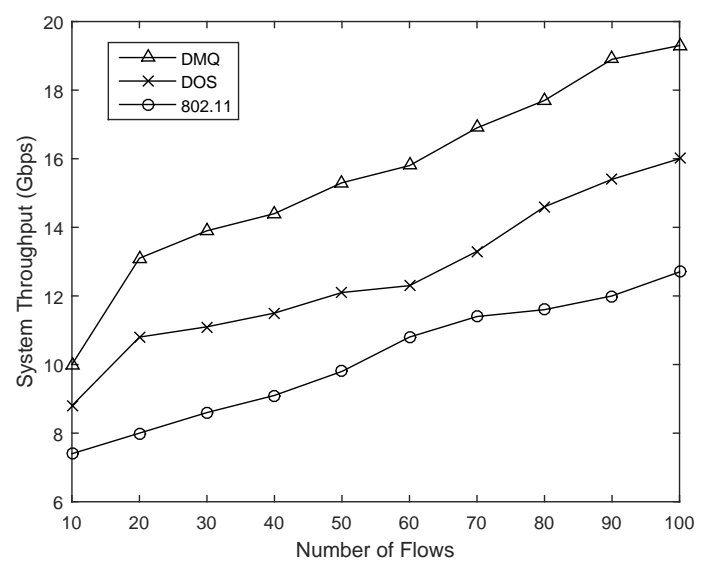

Figure 3: Number of flows on throughput performance.

well as default 802.11 random access. We recorded the elapsed time between moment when first packet of the whole network was sent at transport layer and moment when last packet of the whole network was successfully received at one base station. Then we calculated the overall system throughput for each scheme. We recorded throughput performance of each scheme for different number of flows. Each of the experiment was repeated 10 times and the average results were taken. Finally, we plotted the comparison results shown in Figure 3.

We have the following observations:

- Our proposed DMQ achieves the best result in system throughput performance among all of the three schemes. It achieves an average of $25 \%$ throughput gain over DOS and $40 \%$ over 802.11. Thanks to the coarseto-fine hierarchical structure of DMQ, the scheduler is able to make best use of the available contention window values and assigns them to flows according to both their contention and QoS priorities. Thus the contention between different flows with different priorities can be reduced to minimum.

- Our proposed DMQ is better suited for practical environment. From Figure 3, we see that DOS does not achieve the best performance although it is theoretically proved to be optimal. This is due to the fact that DOS relies on highly accurate past channel estimation to determine current scheduling scheme. However, in practical environment, it is difficult to achieve such an accurate channel estimation in real time. This makes DMQ perform much better than DOS.

\subsubsection{Effects of Number of Packets in Each Flow on Throughput Performance}

In this experiment, we randomly add 50 flows in the system. Then, we vary the number of packets for each flow from 10 to 100 . Other setups remain the 


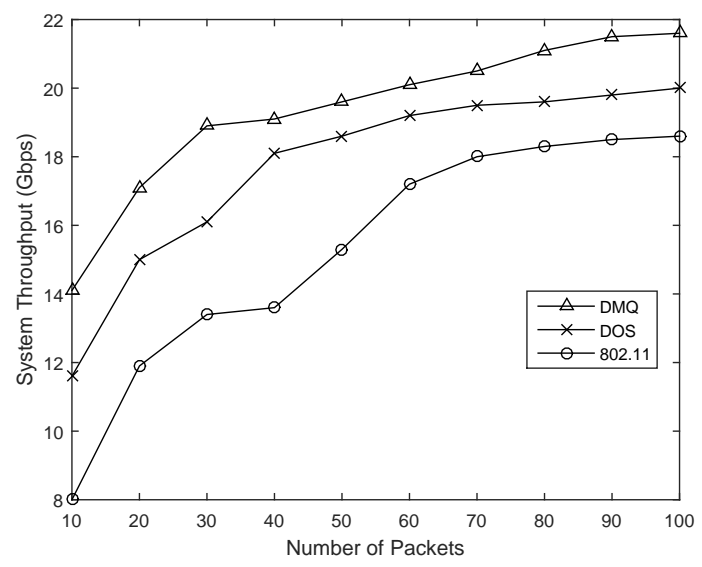

Figure 4: Number of packets in each flow on throughput performance.

same as described in Section 5.2.1. As previously, we recorded the elapsed time between moment when first packet of the whole network was sent at transport layer and moment when last packet of the whole network was successfully received at one base station. The performance comparison is shown in Figure 4.

It clearly shows that:

- Our DMQ outperforms both DOS and default 802.11 scheduling schemes in terms of throughput performance. DMQ can achieve more than $20 \%$ gain over DOS and $40 \%$ gain over 802.11 as the number of packets increases.

- The general trend is that when the number of packets in each flow increases, the overall system throughput also increases.

- As the number of packets increases, the increasing trend of system throughput tends to slow down at the end. This is because the system is more and more approaching its capacity as more and more packets are injected into the system.

\subsubsection{Effects of Antenna Beamwidth on Throughput Performance}

One of the major difference between omni-directional antenna and directional antenna is the beamwidth. In directional antenna patterns, the beamwidth is the angle between half-power $(-3 \mathrm{~dB})$ points and the main lobe. Therefore, beamwidth is an important factor that affects the performance of directional antennas. In this experiment, we study the effects of antenna beamwidth on system throughput. We set the number of flows to 50 and randomly assigns them to base station pairs in the system. In each flow, we set the number of packets to 50 . Then, we vary the beamwidth from $5^{\circ}$ to $50^{\circ}$. As previously, we recorded the elapsed time between moment when first packet of the whole network was sent at transport layer and moment when last packet of the whole 


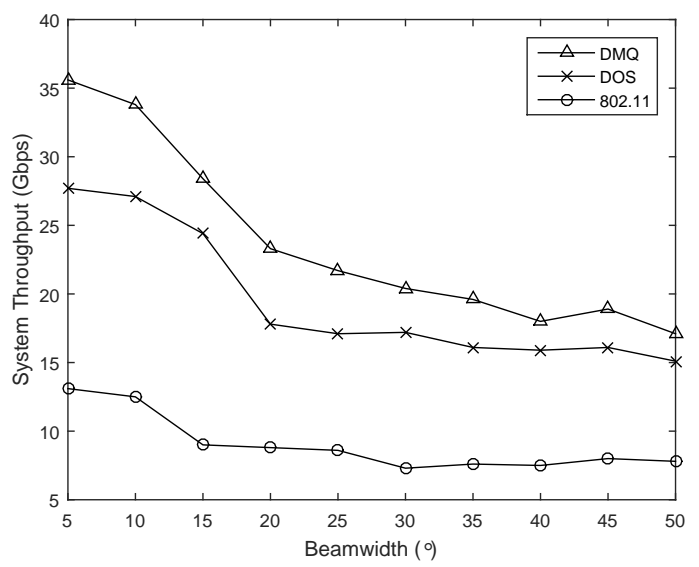

Figure 5: Antenna beamwidth on throughput performance.

network was successfully received at one base station. We collect the experiment data and compare the result in Figure 5.

We observe that:

- Our proposed DMQ achieves the best throughput performance among all the three schemes compared. It can achieve a maximum of $30 \%$ gain over DOS and even triple the performance of 802.11.

- The system throughput decreases as the beamwidth increases. This is because smaller beamwidth means a narrower antenna pattern between half-power and main lobe, which would consequently make the wireless signal more concentrated. As a result, the interference between base stations will be reduced, making the whole system less contending. Thus the throughput can be achieved a lot higher.

- When beamwidth keeps increasing, the contention between base stations also increases as more and more base stations become able to detect the signals from neighboring base stations. This leads to the trigger of back-off mechanism, thus reducing the system throughput.

\subsubsection{Effects of Number of Flows on Packet Loss Ratio}

In this experiment, we focus on the relationship between number of flows in the system and its corresponding packet loss ratio. The experiment setup and methodology are similar to the those described in Section 5.2.1. However, instead of recording throughput, here we record the total number of packets that are successfully received by all receiving base stations. Using such information, we are able to calculate the packet loss ratio (PLR) as:

$$
P L R=1-\frac{\text { Total number of packets received }}{\text { Total number of packets sent in the network }} .
$$




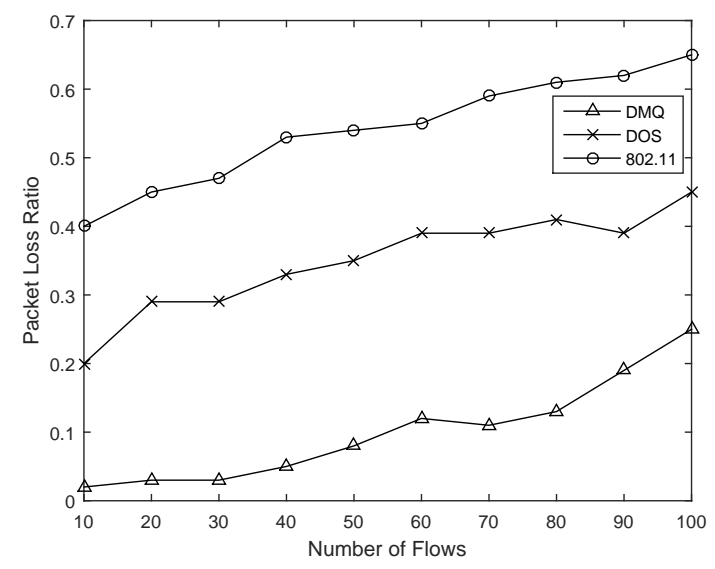

Figure 6: Effects of Number of Flows on Packet Loss Ratio, the less the better.

Again, the experiment was repeated 10 times and the average results were taken. The results of comparison is shown in Figure 6.

We have the following observations:

- Our proposed DMQ achieves the least packet loss ratio which indicates the best performance among the three. It almost can achieve close-to-zero packet loss ratio when the number of flows is small.

- Thanks to CFWM algorithm, DMQ is able to detect and avoid the contention as much as possible, and only loses half of packets DOS has lost and a quarter of packets conventional 802.11 has lost.

- The packet loss ratio of all three schemes tends to increase as the number of flows increases. This is because when number of flows increases, the probability of contention between different flows is also increasing, which leads to the increase of packet loss ratio. Although increasing, the proposed DMQ always keeps packet loss ratio much lower than others.

\section{Conclusion}

In this paper, we have considered the problem of backhaul scheduling to maximize the system throughput while having flows' QoS requirements satisfied in the mmWave backhaul network. The formulated problem is NP-hard, thus we proposed the DMQ scheduling algorithm, where it is designed to achieve the spatial reuse while reducing interference and channel contention between neighbors in a coarse-to-fine two-phase fashion. The QoS aware priority is exploited in the algorithm to provide better QoS guarantees for flows. We conducted extensive experiments and results have shown that our proposed algorithm is able to achieve much higher system throughput and much lower packet loss ratio than other existing schemes under different criteria. 


\section{Acknowledgment}

This work was supported in part by National Science Foundation under Grant ECCS-1509212 and NSFC under grant 61529101.

\section{References}

[1] "Qualcomm data challenge," accessed March 2015, https://www.qualcomm.com/documents/rising-meet-1000x-mobile-datachallenge.

[2] R. Baldemair, T. Irnich, K. Balachandran, E. Dahlman, G. Mildh, Y. Seln, S. Parkvall, M. Meyer, and A. Osseiran, "Ultra-dense networks in millimeter-wave frequencies," IEEE Communications Magazine, vol. 53, no. 1, pp. 202-208, January 2015.

[3] M. N. Islam, A. Sampath, et. al., "Wireless backhaul node placement for small cell networks," in 2014 48th Annual Conference on Information Sciences and Systems (CISS) (Princeton, USA), Mar. 19-21, 2014, pp. 1-6.

[4] U. Siddique, H. Tabassum, E. Hossain, and D. I. Kim, "Wireless backhauling of $5 \mathrm{~g}$ small cells: challenges and solution approaches," Wireless Communications, IEEE, vol. 22, no. 5, pp. 22-31, 2015.

[5] X. Ge, H. Cheng, M. Guizani, and T. Han, "5g wireless backhaul networks: challenges and research advances," Network, IEEE, vol. 28, no. 6, pp. 6-11, 2014.

[6] T. S. Rappaport, J. N. Murdock, F. Gutierrez, "State of the art in 60-GHz integrated circuits and systems for wireless communications," Proceedings of the IEEE, vol. 99, no. 8, pp. 1390-1436, Aug. 2011.

[7] ECMC TC48, ECMA standard 387, "High rate $60 \mathrm{GHz}$ PHY, MAC and HDMI PAL," Dec. 2008.

[8] IEEE 802.15.3 Working Group, "Part 15.3: Wireless Medium Access Control (MAC) and Physical Layer (PHY) Specifications for High Rate Wireless Personal Area Networks (WPANs)," IEEE Unapproved Draft Std P802.15.3c/D10, Jun. 2009.

[9] IEEE 802.11ad Working Group, "Part 11: Wireless LAN Medium Access Control (MAC) and Physical Layer (PHY) Specifications-Amendment 5: Enhancements for Very High Throughput in the 60GHz Band," IEEE Unapproved Draft Std P802.11ad/D1.0, 2010.

[10] J. Wang, Z. Lan, C. Pyo, T. Baykas, C. Sum, M. Rahman, J. Gao, R. Funada, F. Kojima, and S. Kato, "Beam codebook based beamforming protocol for multi-Gbps millimeter-wave WPAN systems," IEEE J. Selected Areas in Communications, vol. 27, no. 8, pp. 1390-1399, Oct. 2009. 
[11] C. Sum, Z. Lan, R. Funada, J. Wang, T. Baykas, M. A. Rahman, and H. Harada, "Virtual Time-Slot Allocation Scheme for Throughput Enhancement in a Millimeter-Wave Multi-Gbps WPAN System," IEEE J. Sel. Areas Commun., vol. 27, no. 8, pp. 1379-1389, Oct. 2009.

[12] C.-S. Sum, Z. Lan, M. A. Rahman, et. al., "A multi-Gbps millimeter-wave WPAN system based on STDMA with heuristic scheduling," in Proc. IEEE GLOBECOM (Honolulu, HI), Nov. 30-Dec. 4, 2009, pp. 1-6.

[13] L. X. Cai, L. Cai, X. Shen, and J. W. Mark, "REX: a Randomized EXclusive Region based Scheduling Scheme for mmWave WPANs with Directional Antenna," IEEE Trans. Wireless Commun., vol. 9, no. 1, pp. 113121, Jan. 2010.

[14] J. Qiao, L. X. Cai, and X. Shen, et. al., "Enabling multi-hop concurrent transmissions in $60 \mathrm{GHz}$ wireless personal area networks," IEEE Transactions on Wireless Communications, vol. 10, no. 11, pp. 3824-3833, Nov. 2011.

[15] Qiao, Jian and Cai, Lin X and Shen, Xuemin and Mark, Jon W, "STDMAbased scheduling algorithm for concurrent transmissions in directional millimeter wave networks," IEEE International Conference on Communications (ICC), pp. 5221-5225, 10-15 June 2012.

[16] A. Mesodiakaki, F. Adelantado, L. Alonso, and C. Verikoukis, "Energyefficient user association in cognitive heterogeneous networks," Communications Magazine, IEEE, vol. 52, no. 7, pp. 22-29, 2014.

[17] J. Qiao, X. S. Shen, J. W. Mark, Q. Shen, Y. He, L. Lei, "Enabling deviceto-device communications in millimeter-wave $5 \mathrm{G}$ cellular networks," IEEE Communications Magazine, vol. 53, no. 1, pp. 209-215, January 2015.

[18] S. Y. Geng, J. Kivinen, X. W. Zhao, and P. Vainikainen, "Millimeter-wave propagation channel characterization for short-range wireless communications," IEEE Trans. Veh. Technol., vol. 58, no. 1, pp. 3-13, Jan. 2009.

[19] I. K. Son, S. Mao, M. X. Gong, and Y. Li, "On frame-based scheduling for directional mmWave WPANs," in Proc. IEEE INFOCOM (Orlando, FL), Mar. 25-30, 2012, pp. 2149-2157.

[20] D. Zheng, W. Ge and J. Zhang, "Distributed Opportunistic Scheduling for Ad Hoc Networks With Random Access: An Optimal Stopping Approach," IEEE Trans. Information Theory, vol.55, no.1, pp.205-222, Jan. 2009.

[21] M. X. Gong, R. Stacey, D. Akhmetov, and S. Mao, "A directional csma/ca protocol for mmwave wireless pans," in IEEE Wireless Communications and Networking Conference (WCNC), 2010. IEEE, 2010, pp. 1-6. 
[22] M. X. Gong, D. Akhmetov, R. Want, and S. Mao, "Directional csma/ca protocol with spatial reuse for mmwave wireless networks," in IEEE Global Telecommunications Conference (GLOBECOM 2010), 2010. IEEE, 2010, pp. 1-5.

[23] K. Lee, Y. Kim, K. Shin, J. Kwon, and C.-H. Kang, "A dynamic cap allocation algorithm using directional antennas in ieee 802.15. 3c wpans," in 13th International Conference on Advanced Communication Technology (ICACT), 2011. IEEE, 2011, pp. 296-299.

[24] Akdeniz, Mustafa Riza and Liu, Yuanpeng and Samimi, Mathew K and Sun, Shu and Rangan, Sundeep and Rappaport, Theodore S and Erkip, Elza, "Millimeter wave channel modeling and cellular capacity evaluation," IEEE Journal on Selected Areas in Communications, vol. 32, pp. 11641179, June 2014.

[25] Toyoda, I., T. Seki, and K. Iiguse, "Reference antenna model with side lobe for TG3c evaluation." IEEE document, pp. 802-15, 2006.

[26] I. Toyoda, T. Seki, K. Iigusa, H. Sawada, Y. Fujita, A. Miura, and N. Orihashi, "Reference antenna model with sidelobe level for tg3c evaluation," IEEE, vol. 802, pp. 06-0474, 2006.

[27] NS-3, "Ns-3," 2015, [accessed 13-October-2015]. [Online]. Available: https://www.nsnam.org/ 
Jiade Li received his B.E from University of Science and Technology of China, Hefei, China in 2010, and M.S from Chinese Academy of Sciences in 2013. He is currently a Ph.D. student in Electrical \& Computer Engineering at University of Florida. His research interests are network congestion control and network performance improvements.

Yun Zhu received his B.E from University of University of Science and Technology of China, Hefei, China in 2013. He is currently a Ph.D. student in Electrical \& Computer Engineering at University of Florida. His research interests are focused on issues of next generation mobile networks.

Dapeng Oliver Wu (S'98--M'04--SM'06--F'13) received B.E. in Electrical Engineering from Huazhong University of Science and Technology, Wuhan, China, in 1990, M.E. in Electrical Engineering from Beijing University of Posts and Telecommunications, Beijing, China, in 1997, and Ph.D. in Electrical and Computer Engineering from Carnegie Mellon University, Pittsburgh, PA, in 2003. He is a professor at the Department of Electrical and Computer Engineering, University of Florida, Gainesville, FL. His research interests are in the areas of networking, communications, signal processing, computer vision, machine learning, smart grid, and information and network security. Currently, he serves as an Associate Editor for IEEE Transactions on Circuits and Systems for Video Technology and IEEE Transactions on Signal and Information Processing over Networks. He is the founder of IEEE Transactions on Network Science and Engineering. He was the founding Editor-in-Chief of Journal of Advances in Multimedia between 2006 and 2008, and an Associate Editor for IEEE Transactions on Wireless Communications and IEEE Transactions on Vehicular Technology between 2004 and 2007. He is also a guest-editor for IEEE Journal on Selected Areas in Communications (JSAC), Special Issue on Cross-layer Optimized Wireless Multimedia Communications. He has served as Technical Program Committee (TPC) Chair for IEEE INFOCOM 2012, and TPC chair for IEEE International Conference on Communications (ICC 2008), Signal Processing for Communications Symposium, and as a member of executive committee and/or technical program committee of over 80 conferences. He has served as Chair for the Award Committee, and Chair of Mobile and wireless multimedia Interest Group (MobIG), Technical Committee on Multimedia Communications, IEEE Communications Society. He was an elected member of Multimedia Signal Processing Technical Committee, IEEE Signal Processing Society from Jan. 1, 2009 to Dec. 31, 2012. He is an IEEE Fellow. 
Jiade Li

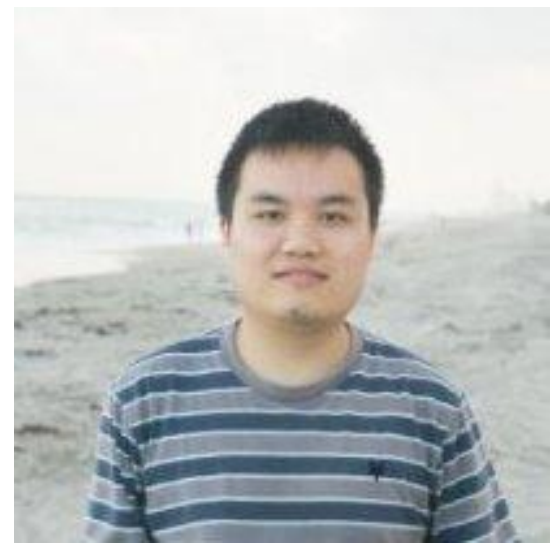

Yun Zhu

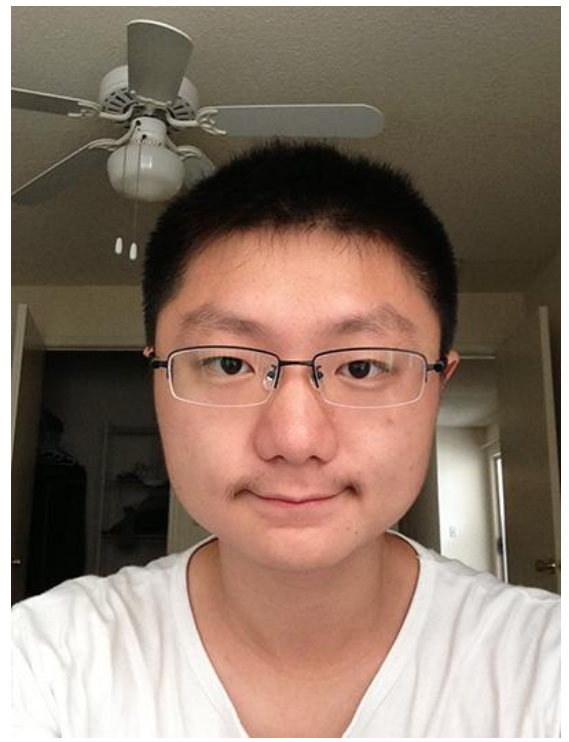

Dapeng Wu

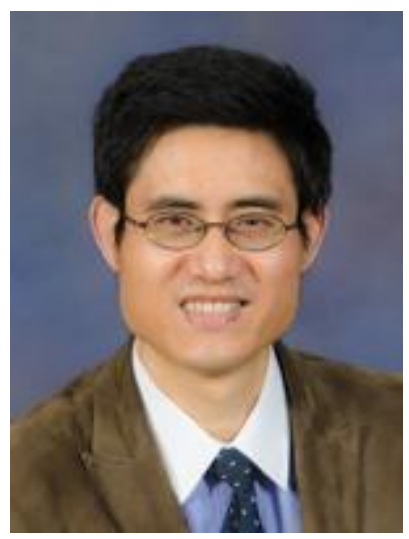

